\title{
Dürre, Waldbrände, gravitative Massenbewegungen und andere klimarelevante Naturgefahren
}

Thomas Glade, Peter Hoffmann, Kirsten Thonicke

12.1 Dürre - 112

12.1.1 Einordnung vergangener Ereignisse - 112

12.1.2 Projektionen - 113

12.2 Waldbrand - 113

12.2.1 Bestandsaufnahme - 113

12.2.2 Projektionen - 114

12.2.3 Perspektiven -114

12.3 Gravitative Massenbewegungen - 115

12.3.1 Felsstürze - 115

12.3.2 Muren - 116

12.3.3 Rutschungen - 116

12.4 Kryosphäre - 117

12.4.1 Auftauender Permafrost - 117

12.4.2 Glaziale Systeme - 118

12.4.3 Schneelawinen - 118

12.5 Ausblick - 118

12.6 Kurz gesagt - 119

Literatur - 119 


\subsection{Dürre}

Neben meteorologischen und hydrologischen Risiken wie Stürmen und Überschwemmungen gehören Dürren zur Kategorie klimatologischer Extremereignisse. Weltweit gesehen beträgt ihr Anteil an allen registrierten Extremereignissen etwa $15 \%$ (Münchner Rückversicherungs-Gesellschaft 2012). Im WMOReport 2013 (WMO 2014) erfolgt eine detailliertere Differenzierung dieser Ereignisklasse. Dabei fallen - weltweit gesehen - noch 6\% in die Kategorie der Dürren. In Deutschland bzw. Europa liegt der Wert etwas darunter. Einen Zustand der atmosphärischen Zirkulationsverhältnisse in der unteren Troposphäre, bei dem es großräumig zu einem außergewöhnlich hohen Wasserdefizit im Boden kommt und bei dem eine ausreichende Wasserversorgung der Pflanzen nicht gewährleistet ist, bezeichnet man als meteorologische Dürre. Witterungssituationen, die lang anhaltend hohe Temperaturen, verhältnismäßig wenig Regen sowie hohe Einstrahlung begünstigen, können das Gleichgewicht zwischen Niederschlag und Verdunstung und damit den Wasserhaushalt erheblich stören. Ein solcher in unregelmäßigen Abständen wiederkehrender Zustand kann verschieden stark ausgeprägt sein und prinzipiell an jedem Ort der Erde auftreten. Zudem werden noch zwei weitere dürreähnliche Zustände unterschieden: Anders als die oben beschriebene Dürre werden diese nicht an klimatologischen Variablen festgemacht, sondern durch hydrologische oder landwirtschaftliche Variablen charakterisiert. Letztlich bestimmen auch die vorherrschenden sozioökonomischen Faktoren der betroffenen Region das Maß der Auswirkungen und Schäden - z. B. die Bevölkerungsdichte, die Landnutzung oder auch die Industrialisierung. Je nach Dauer derartiger Witterungssituationen können, beispielsweise durch das Absinken des Grundwasserspiegels und das Austrocknen kleinerer Binnengewässer, viele Bereiche unseres täglichen Lebens in Mitleidenschaft gezogen werden.

\subsubsection{Einordnung vergangener Ereignisse}

Meteorologische Ursachen, welche die Entwicklung sommerlicher Hitze- und Trockenperioden in Deutschland hervorrufen, werden durch die großräumige atmosphärische Zirkulation über Europa bestimmt. Die Verlagerungsgeschwindigkeit und Intensität atmosphärischer Wellen, die in mittleren Breiten unser Wettergeschehen maßgeblich prägen, werden durch viele Faktoren gesteuert, die sich nur im globalen Kontext erfassen lassen. Diese periodisch den Globus in höheren Atmosphärenschichten umspannenden Strukturen bestimmen wesentlich die Druck- und Temperaturverteilung und verlagern sich parallel zum Äquator zeitlich von West nach Ost. Sie können aus bis zu acht Wellenbergen und -tälern bestehen, und durch Überlagerungserscheinungen (Petoukhov et al. 2013) können sich stabile großräumige Wettersituationen einstellen (DWD 2004). So gelangten z. B. 2003 heiße und trockene Luftmassen aus der Sahara nach Mitteleuropa, die zu Rekordwerten in Europa und Deutschland führten, die bis dato als sehr unwahrscheinlich galten (Schär et al. 2004). - Abb. 12.1 zeigt die großräumigen Unterschiede in Temperatur und Niederschlag während des Sommers 2003 in
Bezug auf die Referenzperiode 1981-2010. Diese Konfiguration der atmosphärischen Zirkulation führte in weiten Teilen Europas zu Temperaturen, die den klimatologischen Durchschnitt um $1-4{ }^{\circ} \mathrm{C}$ übertrafen (- Abb. 12.1). In Deutschland lagen die Abweichungen zur Klimanormalperiode (1961-1990) für Nordund Mitteldeutschland zwischen +3 und $+4{ }^{\circ} \mathrm{C}$ (DWD 2014), im südlichen Teil Deutschlands deutlich darüber.

Anders als bei Extremereignissen wie Hochwasser, Stürmen und lokalen Unwettern besitzen Dürreperioden häufig großräumige Flächenausdehnungen. Sie können sowohl die Wirtschaft als auch langfristig das Wohlbefinden der Menschen stärker beeinträchtigen als andere Ereignisse (WMO 2012). Selbst stärkere Niederschläge vor oder nach Dürreperioden verringern häufig nicht das Schadenspotenzial: Wie das Jahr 2011 gezeigt hat, konnten auch überdurchschnittliche Regenmengen im Winter und Sommer das Defizit im März bis Mai sowie Oktober und November nicht kompensieren. Die Auswirkungen von Dürreperioden sind nicht nur großflächig, sondern häufig auch lang anhaltend.

Perioden mit extremer Trockenheit sind also nicht nur auf die Sommermonate begrenzt, sondern können auch im Frühjahr und Herbst spürbare Folgen hinterlassen. Ihre Ausprägung wird vor allem durch die Stabilität von Hochdruckwetterlagen über Mitteleuropa bestimmt. Bei längerem Andauern verdunstet mehr Wasser aus dem Boden, als ihm durch Regen zugeführt wird.

Obwohl sich in einem wärmeren Klima potenziell mehr Wasser in Dampfform in der Atmosphäre befindet, entscheiden letztlich die großräumige Zirkulation und die niederschlagsbildenden Prozesse darüber, ob das verfügbare Angebot an Wasser tatsächlich als Niederschlag zum Boden gelangt oder in der Atmosphäre verbleibt. Auswertungen von Stationsdaten in Deutschland von 1951 bis 2006 bestätigen, dass die relative Luftfeuchtigkeit trotz einer Zunahme der spezifischen Luftfeuchtigkeit abnimmt (Hattermann et al. 2013). Dabei wird der Masseanteil von Wasserdampf in feuchter Luft zwar größer, zugleich steigt jedoch der Abstand zwischen dem Dampfdruck des Wassers und dem durch die höhere Temperatur gestiegenen Sättigungsdampfdruck. Dadurch entsteht eine höhere Schwelle bei der Wolken- und Niederschlagsbildung, die örtlich zu längeren niederschlagsfreien Perioden und im Sommer zu niedrigeren Abflusswerten führen kann (Krysanova et al. 2008). So fällt bei der Betrachtung von Temperatur- und Niederschlagsaufzeichnungen (DWD 2010) - gemittelt über Deutschland für die Sommermonate Juni bis August - auf, dass sich genau die Jahre abheben (1983 und 1947), die in der Vergangenheit durch ein extremes Wasserdefizit geprägt waren.

Statistische Analysen der langen Beobachtungsreihen (1901-2003) für Deutschland zeigen, dass Sommeranomalien wie im Jahr 2003 mit einer Eintrittswahrscheinlichkeit von weniger als $0,01 \%$ außerordentlich selten sind (Schönwiese und Janoschitz 2005). Das entspricht in etwa einem Ereignis pro 10.000 Jahren. Betrachtet man die Wahrscheinlichkeit für derartige Extremereignisse allerdings nach dem Extremsommer 2003, ergibt sich nach heutigem Wissen ein sehr viel kürzeres Wiederkehrintervall: nämlich nur von etwa 450 Jahren (Schönwiese und Janoschitz 2005). Derartige Hitzewellen und Dürreperioden werden jedoch unter einem wärmeren Klima wahrscheinlicher (IPCC 2013). 


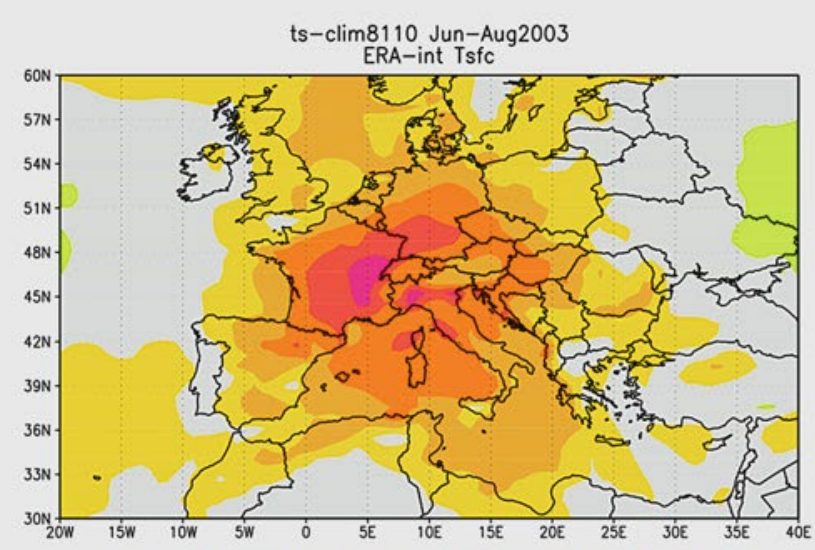

a

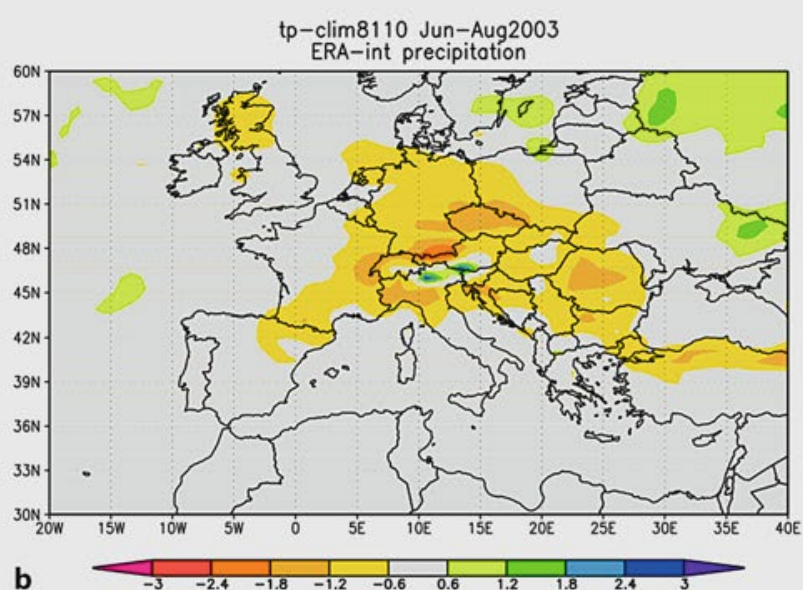

- Abb. 12.1 Großräumige Verteilung der Temperatur- (a in ${ }^{\circ} \mathrm{C}$ ) und Niederschlagsabweichungen $(\mathbf{b}$ in $\mathrm{mm} / \mathrm{d}$ ) über Europa während des Sommers 2003 (Juni bis August) bezogen auf den Referenzzeitraum 1981-2010. (Climate Explorer o.J.)

\subsubsection{Projektionen}

Aus den globalen Klimaprojektionen des Fünften Sachstandsberichts des Weltklimarats (IPCC 2013) geht hervor, dass sich an der Häufigkeit blockierender Wetterlagen über Europa nur wenig ändern wird. Solche Wetterlagen sind besonders wichtig für die Ausbildung von Dürren. Allerdings gibt es Anzeichen dafür, dass sich sowohl die Andauer als auch die Intensität derartiger Zustände in der Atmosphäre verstärken könnten (Masato et al. 2013). Gesicherte Aussagen darüber sind jedoch nicht möglich. Allerdings zeigen regionalisierte Klimaprojektionen unter Annahme verschiedener Emissionsszenarien für Europa eine $\mathrm{Zu}$ nahme klimatischer Extreme: Die Zahl der Hitzewellen steigt, und die Trockenphasen dauern länger. Die stärkste Ausprägung dieser Größen macht das Emissionsszenario RCP8.5 über Mitteleuropa sichtbar (Jacob et al. 2013). Primär allerdings liegt die größte Gefährdung in Südeuropa - so zeigen es auch frühere Szenarien (Fischer und Schär 2010) -, da die Effekte einer Kombination von zunehmender Hitze und Trockenheit dort noch größere Folgen haben als in Mitteleuropa.

Aber auch für Teile Ostdeutschlands zeigen Klimarechnungen eine Zunahme der sommerlichen Wasserknappheit (Jacob et al. 2013). Diese Situation begünstigt die Entwicklung von Dürreperioden, da sich zudem der mittlere Niederschlag im Sommer verringert. Die zugrunde liegenden Modelle projizieren einen Rückgang um $10 \%$ bis zur Mitte des Jahrhunderts (2021-2050) und um $20 \%$ bis zum Ende des Jahrhunderts (2071-2100). Die Änderungen beziehen sich dabei auf die Referenzperiode 19712000 und sind im Westen stärker als im Osten, da dort die mittlere Niederschlagssumme höher liegt.

Eine generelle Bewertung der in Deutschland betriebenen regionalen Klimamodelle (CCLM, Remo, STARS, WETTREG) konnte bislang nicht in zufriedenstellender Art und Weise umgesetzt werden. Vor allem die zukünftige Entwicklung des Niederschlags ist mit großen Unsicherheiten behaftet. Daher stellt jede einzelne regionale Klimasimulation ein mögliches Szenario für die zukünftige Entwicklung dar.
Ob sich die Häufigkeit und das Ausmaß von Dürren und dürreähnlichen Zuständen in Deutschland und Mitteleuropa zukünftig verändern, wird ausschließlich durch Einflussfaktoren auf der globalen Skala bestimmt. Die dafür verantwortlichen Witterungsverläufe über Mitteleuropa resultieren aus der Verstärkung troposphärisch angeregter planetarer Wellen mit niedriger Verlagerungsgeschwindigkeit. Sie können teils länger als eine Woche quasi stationär verweilen. Auch wenn sich diese in ihrer Häufigkeit nicht wesentlich ändern werden, so könnten doch die Folgen des Rekordsommers 2003 bei längerer Andauer übertroffen werden. Bisherige kurzfristigere Trockenperioden können sich dann zukünftig zu länger anhaltenden Dürren ausweiten.

\subsection{Waldbrand}

\subsubsection{Bestandsaufnahme}

Feuerregime, also Muster, die das Zusammenspiel zwischen Feuergefährdung, Entzündungen und Flächengröße beschreiben, sind ständigen Veränderungen und Anpassungen unterworfen, die durch Klimaschwankungen, Vegetationsdynamik und auch durch den Menschen hervorgerufen werden. Brände treten in Deutschland vornehmlich als Waldbrände auf oder werden gezielt zur Landschaftspflege und zum Erhalt geschützter Biotope gelegt, z. B. auf Heideflächen. Waldbrände sind in Deutschland von Natur aus sehr selten, da es keine Zündung oder Selbstentzündung durch Sonneneinstrahlung gibt, wie man dies häufig in den Mittelmeerregionen beobachten kann. Die übergeordnete natürliche Waldbrandursache ist in Deutschland der Blitzschlag (Müller 2009). Aufgrund der besonderen Bodeneigenschaften und des Übergangs zum Kontinentalklima besteht im Nordosten Deutschlands eine höhere Waldbrandgefahr als in anderen Regionen des Landes.

Das gemäßigte Klima, die stark fragmentierte Landschaft, aber auch das effektive Waldbrand-Monitoringsystem bewirken, dass bisher nur in extrem trockenen Jahren Waldbrände auftre- 
ten, wobei bis zu 2000 ha Wald verbrennen - bei einem gleichzeitig seit drei Jahrzehnten rückläufigen Trend der jährlichen Waldbrandflächen (BLE 2011): Vor allem das Monitoring hat sich verbessert, da inzwischen automatisierte Detektionssysteme eingesetzt werden. Allerdings hat über den gleichen Zeitraum die Klimavariabilität zugenommen, was die zunehmende Anzahl an Tagen mit hoher Waldbrandwarnstufe (Wittich et al. 2011) zeigt. Weiterhin wurde analysiert, dass extrem hohe Wandbrandgefahr in kürzeren Intervallen auftritt (Wastl et al. 2012). Einen ebenfalls seit 1958 signifikanten, zunehmenden Trend fanden Lavalle et al. (2009) in der Intensität der Feuersaison, d.h. größere Feuergefahr bei gleicher Saisonlänge, in Mittel- und Nordostdeutschland. Obwohl also die klimatisch bedingte Waldbrandgefahr steigt, kompensieren Sicherungsmaßnahmen dies derzeit noch. Weiterhin ist festzuhalten, dass sich die geplante Waldentwicklung und -strukturveränderung weg von großflächigen Monokulturen hin zu diverseren Waldbeständen bewegt hat, wodurch die potenzielle Waldbrandgefahr reduziert ist (Müller 2009).

\subsubsection{Projektionen}

Inwiefern sich dieser Trend zunehmender Feuergefährdung in der Zukunft fortsetzen wird, hängt nicht nur vom projizierten Klimawandel selbst $\mathrm{ab}$, sondern auch von Veränderungen im Waldwachstum und den physiologischen Prozessen von Pflanzen. Offenere, stärker mit Gräsern bewachsene Wälder können bei gleicher klimatischer Gefährdung die Feuerausbreitung begünstigen, dichte Wälder ohne Grasunterwuchs dagegen verringern. Zusätzlich muss natürlich der veränderte Umgang des Menschen mit der Waldwirtschaft beachtet werden. Besonders die Qualität mit der Feuchte und Lage und die Quantität mit der Menge und Dimension des Brennmaterials bestimmen maßgeblich die Waldbrände. Jedoch kann auch ein höherer atmosphärischer $\mathrm{CO}_{2}$-Gehalt die Wassernutzungseffizienz der Wälder und damit die Wasserspeicherung im Boden erhöhen. Dieser sogenannte $\mathrm{CO}_{2}$-Düngungseffekt kann die Produktivität der Wälder steigern ( Kap. 17) und dabei möglicherweise indirekt die Waldbrandgefahr reduzieren. Daher muss zwischen Projektionen der Waldbrandgefahr und der Veränderung der Feuerregime unterschieden werden, die mithilfe von gekoppelten VegetationFeuer-Modellen simuliert werden können.

Da Waldbrände im Mittelmeerraum ein größeres Problem darstellen, sind vor allem Projektionen der Waldbrandgefahr und der Veränderung der Feuerregime für diese Region erstellt worden (Amatulli et al. 2013; San-Miguel-Ayanz et al. 2013). Aber auch für Deutschland sind entsprechende Projektionen aus europäischen, nationalen oder regionalen Studien verfügbar, die auf globale oder regionale Klimaprojektionen des Vierten Sachstandberichts des IPCC zurückgreifen. Die Emissionsszenarien SRES A2, A1B und A1FI wurden zugrunde gelegt, um die stärksten Veränderungen im zukünftigen Waldbrandrisiko zu erfassen. Am häufigsten wurde der kanadische Waldbrandindex FWI (fire weather index, van Wagner 1987) für Projektionen auf nationaler (Wittich et al. 2011) und europäischer Ebene (Lavalle et al. 2009) verwendet. Auch der Deutsche Wetterdienst benutzt für das Monitoring der aktuellen Waldbrandgefahr zunehmend den FWI, auch wenn frühere Arbeiten auf deutsche Indizes zurückgegriffen haben (Badeck et al. 2004). Neuere methodische Untersuchungen des FWI haben jedoch darauf verwiesen, dass berechnete Risikoveränderungen unterschätzt werden könnten oder die geografische Verteilung gefährdeter Gebiete ungenau sein könnte, wenn Tagesmittelwerte statt Tagesmaxima für die Berechnung herangezogen werden. Daher sollten für entsprechende Zukunftsprojektionen Tagesmaxima verwendet werden (Bedia et al. 2013).

Generell ist das Bild der projizierten Veränderungen zukünftiger Waldbrände sehr heterogen und qualitativ unterschiedlich. Dies ist auf Unsicherheiten aus der Klimamodellierung zurückzuführen. Berechnungen, die mehrere regionale Klimasimulationen als Eingangsgrößen für den FWI verwenden, zeigen eine Zunahme des Waldbrandrisikos für die Mitte oder das Ende des 21. Jahrhunderts, beinhalten aber auch Ergebnisse, die eine Reduzierung des Risikos projizieren (- Tab. 12.1). Ein zunehmendes Waldbrandrisiko bedeutet, dass größere Gebiete betroffen sind, dass die Feuersaison länger dauert und dass es mehr Tage mit extremer Waldbrandgefahr gibt (Lavalle et al. 2009). Aktuellste Projektionen der Waldbrandgefahr sind im Klimaatlas des Deutschen Wetterdienstes zusammengefasst (www.deutscherklimaatlas.de/forstwirtschaft).

\subsubsection{Perspektiven}

Projektionen des Waldbrandrisikos beinhalten nur die klimatische Gefährdung, jedoch nicht die Interaktionen zwischen Bestandsstruktur, menschlicher Nutzung und Feuer, die Feuereffekte verstärken oder abschwächen können. Entsprechende Simulationsexperimente mit gekoppelten Vegetation-FeuerModellen, die ebenfalls durch Klimaprojektionen angetrieben wurden, zeigen für Zentraleuropa keine Veränderungen der Feuerregime. Das heißt, weder die Feuerwahrscheinlichkeit noch die verbrannten Flächen zeigen eine signifikante Veränderung. Dies kann durch das verwendete prognostische Feuermodell erklärt werden, das ggf. nichtlineare Veränderungen in den verantwortlichen Prozessen in Zentraleuropa nicht berücksichtigt (Migliavacca et al. 2013a). Dazu könnte eine schnellere Feuerausbreitung zählen, die durch eine Ausdehnung der Grasflächen auf Kosten von Wäldern hervorgerufen würde. Zum anderen kann aber auch der $\mathrm{CO}_{2}$-Düngeeffekt entsprechende Anstiege im klimatischen Waldbrandrisiko ausgleichen und die Veränderungen in den Waldbrandflächen möglicherweise klein halten (Thonicke und Cramer 2006). Zusätzlich ist festzuhalten, dass die menschliche Nutzung das Waldbrandrisiko mitbestimmt.

Während Projektionen des Waldbrandrisikos den klimatischen Rahmen möglicher Veränderungen darstellen, können physiologische Prozesse und konkrete Bestandsstrukturen dazu führen, dass dieser klimatische Rahmen gar nicht zum Tragen kommt. Feuerregime könnten demnach weniger von klimatischen Veränderungen als eher vom Wandel anderer Faktoren abhängig sein, d.h., wenn sich deren Muster verändern, dann verändert sich das tatsächliche Feuerregime. Diese Szenarien zeigen die potenzielle und tatsächliche Zündfähigkeit und Brennbarkeit der vorhandenen Brennmaterialien an. Dies wird sehr 
- Tab. 12.1 Projektionen zukünftiger Waldbrandgefährdungen und Waldbrandregime in Abhängigkeit von regionalen und globalen Klimaprojektionen sowie Emissionsszenarien für das 21. Jahrhundert und deren relative Veränderung im Vergleich zum historischen Zeitraum. Je nach Aspekt der untersuchten Feuerregime und der verwendeten Modelltypen („verwendete Methode“) ergeben sich qualitativ unterschiedliche zukünftige Veränderungen

\begin{tabular}{|c|c|c|c|c|c|c|c|}
\hline \multirow[t]{2}{*}{ Klimamodell } & \multirow{2}{*}{$\begin{array}{l}\text { Emissions- } \\
\text { szenario }\end{array}$} & \multicolumn{2}{|c|}{ Verwendete Methode } & \multirow[t]{2}{*}{ Region } & \multicolumn{2}{|c|}{ Berechnete Veränderung } & \multirow[t]{2}{*}{ Referenz } \\
\hline & & $\begin{array}{l}\text { Feuerrisiko- } \\
\text { index }\end{array}$ & $\begin{array}{l}\text { Feuermo- } \\
\text { dell }\end{array}$ & & Zeitraum & $\begin{array}{l}\text { Veränderung: } \\
\text { starke Zunahme: ++ } \\
\text { leichte Zunahme: + } \\
\text { keine Änderung: } 0 \\
\text { leichte Abnahme: - } \\
\text { starke Abnahme: - - }\end{array}$ & \\
\hline DMI-HIRHAM & SRES A2 & SSR des FWI & & Europa & $\begin{array}{l}2071-2100 \text { vs. } \\
1961-1990\end{array}$ & + & $\begin{array}{l}\text { Lavalle et al. } \\
\text { (2009) }\end{array}$ \\
\hline $\begin{array}{l}\text { CLM, REMO, STAR, } \\
\text { WETTREG** }\end{array}$ & SRES A1B & FWI & & $\begin{array}{l}\text { Deutsch- } \\
\text { land }\end{array}$ & $\begin{array}{l}2021-2050 \text { vs. } \\
1971-2000\end{array}$ & $\begin{array}{l}++ \text { (STAR) } \\
++(\text { CLM }) \\
+ \text { (WETTREG) } \\
\text { - (REMO) }\end{array}$ & $\begin{array}{l}\text { Wittich et al. } \\
\text { (2011) }\end{array}$ \\
\hline $\begin{array}{l}\text { KNMI-RACMO2 } \\
\text { METO-HC-Had- } \\
\text { RM3Q0 } \\
\text { DMI-HIRHAM5 }\end{array}$ & SRES A1B & FWI & & Europa & $\begin{array}{l}2071-2100 \text { vs. } \\
1971-2000\end{array}$ & $\begin{array}{l}+(\operatorname{HadRM} 3 \mathrm{Q} 0) \\
+(\text { RACMO2) } \\
-(\text { HIRHAM })\end{array}$ & $\begin{array}{l}\text { FP7-FUME pro- } \\
\text { ject } \triangleright \text { http:// } \\
\text { cordis.europa. } \\
\text { eu/result/ } \\
\text { rcn/54266_ } \\
\text { en.html }\end{array}$ \\
\hline $\begin{array}{l}\text { COSMO-CLM, } \\
\text { ENSEMBLES-RCM }\end{array}$ & $A 1 B$ & FFMC & & Europa & $\begin{array}{l}2031-2050 \text { vs. } \\
1991-2010\end{array}$ & $\begin{array}{l}-(\mathrm{COSMO}) \\
+(\mathrm{RCMs})\end{array}$ & $\begin{array}{l}\text { Cane et al. } \\
\text { (2013) }\end{array}$ \\
\hline \multirow{2}{*}{$\begin{array}{l}\text { KNMI-RACMO2, } \\
\text { ECHAM5-HIRHAM5, } \\
\text { METO-HC-Had- } \\
\text { RM3Q0, ARPEGE-HIR- } \\
\text { HAM5, HadCM3-RCA }\end{array}$} & \multirow[t]{2}{*}{$\mathrm{A} 1 \mathrm{~B}$} & & \multirow[t]{2}{*}{ CLM-AB* } & Europa & $\begin{array}{l}2040-2069 \text { vs. } \\
1961-1990\end{array}$ & 0 & \multirow[t]{2}{*}{$\begin{array}{l}\text { Migliavacca } \\
\text { et al. (2013b) }\end{array}$} \\
\hline & & & & Europa & $\begin{array}{l}2070-2099 \text { vs. } \\
1961-1990\end{array}$ & 0 & \\
\hline $\begin{array}{l}\text { HadCM3, CSIRO2, } \\
\text { PCM }\end{array}$ & $\mathrm{A} 1 \mathrm{FI}$ & & $\begin{array}{l}\text { LPJ-Reg- } \\
\text { FIRM** }^{*}\end{array}$ & $\begin{array}{l}\text { Branden- } \\
\text { burg }\end{array}$ & $1975-2100$ & $\begin{array}{l}0 \text { (ohne } \mathrm{CO}_{2} \text {-Effekt) } \\
- \text { (mit } \mathrm{CO}_{2} \text {-Effekt) }\end{array}$ & $\begin{array}{l}\text { Thonicke und } \\
\text { Cramer (2006) }\end{array}$ \\
\hline
\end{tabular}

stark über die Waldbewirtschaftung gesteuert. Die Vorbeugung, Überwachung und Brandbekämpfung können folglich die Waldbrandgefahr auch im Klimawandel stark beeinflussen.

\subsection{Gravitative Massenbewegungen}

Die Naturgefahren der gravitativen Massenbewegungen beinhalten Prozesse wie Felsstürze, Muren, flach- und tiefgründige Rutschungen sowie andere komplexe Bewegungen (Glade et al. 2005). Diese sind auf verschiedenste Art von klimarelevanten Faktoren abhängig und werden ganz unterschiedlich direkt und indirekt vom Menschen beeinflusst (Klose et al. 2015; Schmidt und Dikau 2004). Gravitative Massenbewegungen treten an vollkommen natürlichen, vom Menschen unbeeinflussten Hängen auf, z. B. im hochalpinen Gebiet, an Hängen von eingeschnittenen Tälern und Schichtstufen in Mittelgebirgen (u. a. Hardenbicker et al. 2001; Terhorst 2001, 2009; Schmidt und Beyer 2001; Finkler et al. 2013; Bock et al. 2013; Garcia et al. 2010; Oeltzschner 1997) oder an Steilküsten (Günther und Thiel 2009; Kuhn und Prüfer 2014). Sie treten aber auch an Böschungen auf, die vom Menschen geschaffen wurden, oder an übersteilten Hängen, etwa in Gebieten, in denen Baugebiete ausgewiesen (Kurdal et al. 2006) oder flächenhafte Flurbereinigungen durchgeführt wurden. Je nach Lokalität sind demzufolge die Dispositionen der Gebiete gegenüber klimatischen und hydrometeorologischen Auslösern komplett unterschiedlich (u. a. Dikau und Schrott 1999; Schmidt und Dikau 2004).

\subsubsection{Felsstürze}

Neben den hier nicht weiter behandelten Erdbeben sind besonders Starkniederschläge Auslöser von Felsstürzen, die häufig durch hydrometeorologische Vorgänge vorbereitet werden (Krautblatter et al. 2010a, 2010b). Hierzu gehört z. B. ein langanhaltender Niederschlag, der die offenen Gesteinsklüfte ausfüllt und dort zu großen Porenwasserdrücken führen kann. Diese können auch durch eine Schneeschmelze im Frühjahr erreicht werden. 
Solche vorbereitenden Faktoren lösen gravitative Massenbewegungen nicht direkt aus, sondern erhöhen die Disposition der entsprechenden stabilitätsbeeinflussenden Variablen. Einen weiteren derartigen Faktor im Hochgebirge stellt der Permafrost dar. Der dauergefrorene Bereich stabilisiert die steilen alpinen Felswände zusätzlich (Haas et al. 2009). Durch die Klimaerwärmung werden bisher steile Gesteinsformationen in einen labilen Zustand versetzt und können sich dann entsprechend aus der Felswand ablösen (Krautblatter und Moser 2009; Krautblatter et al. 2013).

Eine ganz andere Situation ist an den Steilküsten Norddeutschlands zu beobachten (Günther und Thiel 2009; Kuhn und Prüfer 2014). Für deren Stabilität sind wieder die Klüftung des Gesteins und der anzutreffende Porenwasserdruck maßgeblich verantwortlich. Hinzu kommt hier aber auch noch die Wellenwirkung über die Brandung: Sie erodiert die Steilküsten kontinuierlich am Hangfuß, bis die darüber gelagerte Masse so instabil wird, dass sie kollabiert. Diese Grenze zwischen Stabilität des Kliffs und der Bewegungsauslösung kann durch interne Kräfteverschiebungen überschritten werden (verursacht $z$. B. durch die Verwitterung des Gesteins), kann aber auch durch externe Kräfte, beispielsweise über einen Sturm mit sehr hoher Brandung oder über Starkniederschläge, erreicht werden. Die klimatischen und hydrometeorologischen Faktoren beeinflussen folglich langfristig über die Wellenbewegungen und die Ausbildung der Brandungshohlkehlen die Stabilität ganzer Küstenabschnitte, führen aber bei extremen Situationen wie Starkniederschlägen oder einer starken Wellenbrandung auch zur Auslösung der Felsstürze.

Weiterhin treten Felsstürze an künstlichen Geländeanschnitten in vielfältigster Weise auf. Solche Anschnitte entstehen sehr häufig beim Bau der Verkehrsnetze (Straßen oder Eisenbahn, Röhlich et al. 2003) oder beim Hausbau in Hangbereichen. Hier kann es auch zur Auslösung der Felsstürze durch hydrometeorologische Faktoren kommen, die eigentliche Ursache im Sinne eines vorbereitenden Faktors ist jedoch in der anthropogenen Übersteilung zu sehen. Untersuchungen zeigten auch, dass zwischen dem Zeitpunkt solcher Übersteilung und dem eigentlichen Auslösen der Felsstürze viele Jahre, manchmal sogar viele Jahrzehnte liegen können. Dies erschwert die klare Trennung zwischen dem menschlichen Einfluss und den deutlich auf die Klimaänderungen zurückzuführenden Folgewirkungen.

Zusammenfassend ist festzuhalten, dass Felsstürze in den verschiedensten Regionen in Deutschland an natürlichen und künstlich übersteilten Felswänden auftreten (Röhlich et al. 2003). Die klimatischen und hydrometeorologischen Wirkungen sind dabei als vorbereitende Faktoren genauso wichtig wie für die Auslösung an sich (Krautblatter und Moser 2006; Schmidt und Dikau 2004). Eine klare Trennung zwischen den natürlichen und damit klar auf den Klimawandel zu beziehenden Gegebenheiten und den vom Menschen beeinflussten Faktoren ist überaus schwierig.

\subsubsection{Muren}

Muren sind Ströme aus Wasser, Schlamm- und Gesteinsmassen, die sich im Gebirge bergabwärts bewegen (- Abb. 12.2). Die kli-

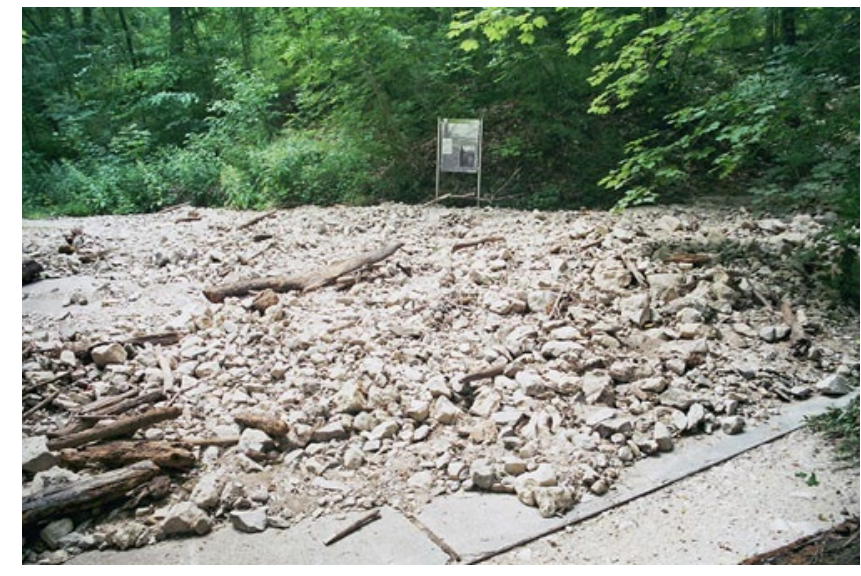

- Abb. 12.2 Murablagerung in Bad Überkingen, Schwäbische Alb. (Rainer Bell)

matischen und hydrometeorologischen Gegebenheiten wirken auch hier als vorbereitende und auslösende Faktoren: Wassergesättigtes Material ist leichter mobilisierbar als trockene Sedimente. Weiterhin spielen auch Vegetationsänderungen für die Muraktivität eine große Rolle. Im Falle einer Rodung oder eines natürlichen Windwurfs können bisher durch die Vegetation geschützte Bereiche bei Sturmereignissen zu potenziellen Quellgebieten von Muren werden. Außerdem können Murverbauungen den Prozessablauf maßgeblich verändern, indem sie beispielsweise die Muren abbremsen oder aufhalten. All dies beeinflusst, wie oft Muren auftreten und wie stark sie sind, und es überlagert mögliche Klimafolgenwirkungen.

Es ist festzustellen, dass sich die durch Klimaereignisse ausgelöste Muraktivität verändert (Damm und Felderer 2013). Dies wurde z. B. auch in dendromorphologischen Untersuchungen erkannt (Schneider et al. 2010), die in den veränderten Jahresringen die Wachstumsveränderungen von Bäumen, verursacht durch die Bewegung der Erdoberflächen, analysieren. Es ist aber nicht eindeutig, welche dieser Veränderungen auf die klimarelevanten Parameter zurückzuführen sind und welche von anderen Einflüssen in welcher Stärke überlagert werden.

\subsubsection{Rutschungen}

Bei Rutschungen werden meist Lockersedimente, aber auch geklüftete Felsmassen auf einer hangparallelen (Translationsrutschung) oder rotationsförmigen Gleitfläche (Rotationsrutschung) hangabwärts transportiert (- Abb. 12.3). Rutschungen treten an natürlichen sowie an künstlich übersteilten Hängen gleichermaßen auf und bewegen sich mit den verschiedensten Geschwindigkeiten: von langsam kriechend bis spontan ausbrechend und extrem schnell. Wichtig ist zu beachten, ob es Neuinitiierungen von Rutschungen sind oder ob es sich um Reaktivierungen bereits früherer Bewegungen handelt. Denn diese reagieren ganz unterschiedlich auf gleiche klimatische und hydro-meteorologische Gegebenheiten.

Auch Rutschungen bereiten sich vor, werden dann ausgelöst, und ihre Bewegung wird durch die Situation am Hang beeinflusst (Schmidt und Dikau 2005) - besonders davon, welche Pflanzen in welchem Alter den Hang bewachsen und durchwurzeln 


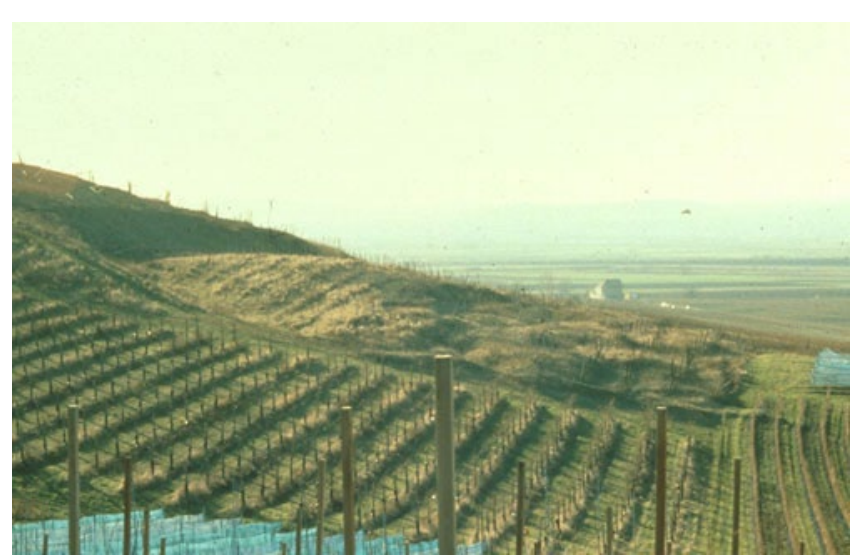

- Abb. 12.3 Rotationsrutschung bei Ockenheim, Rheinhessen. (Thomas Glade)

(Papathoma-Köhle und Glade 2013), wie die Geländeoberfläche geformt ist, wie stark der Boden verwittert ist, welches Gestein ansteht und wie viel Material und Wasser verfügbar sind. Die gleiche Niederschlagsmenge kann also mal Rutschungen auslösen und mal nicht - je nach Situation am Hang.

Viele Untersuchungen zu Rutschungen zeigen auch, dass besonders der Wege- und Siedlungsbau und die veränderten Hangdrainagen einen großen Einfluss auf das Rutschungsverhalten haben (Röhlich et al. 2003; Andrecs et al. 2007). Das gleiche Phänomen trifft auch für den Siedlungsbau zu - wenn auch räumlich weniger ausgedehnt. Wasser wird oberflächig und unterirdisch gesammelt und umgeleitet. Weiterhin werden auch agrarwirtschaftlich genutzte Flächen im Hangbereich sehr häufig von Landwirten dräniert, um die Nutzung zu intensivieren. Alle genannten Aktivitäten verändern die Hanghydrologie, wodurch die Rutschungsaktivität beeinflusst wird.

Natürliche Auslöser von Rutschungen sind neben Erdbeben (z.B. Nepal-Erdbeben, 25.04.15) besonders hydrometeorologische Faktoren. Hierzu zählen lang anhaltende Feuchteperioden oder eine schnelle Schneeschmelze genauso wie Starkregenereignisse (Krauter et al. 2012). Es gibt aber auch Untersuchungen, die eine erhöhte Rutschungsaktivität besonders nach lang anhaltenden Trockenperioden mit anschließenden, von der Stärke eher vernachlässigbaren Niederschlagsereignissen feststellen konnten (Glade und Dikau 2001). Analysen haben gezeigt, dass sich in der Trockenperiode tief greifende Risse im Oberboden bildeten, über die dann der Niederschlag sehr schnell in den Untergrund eindringen konnte und eine Rutschung reaktivierte, obwohl die eigentliche Niederschlagsmenge sehr gering war.

Aus diesen Ausführungen ist ersichtlich, dass es sicherlich einen Zusammenhang zwischen klimatischen Veränderungen und einer daraus resultierenden Rutschungsaktivität gibt (Dehn und Buma 1999; Krauter et al. 2012). Wie jedoch auch aus internationalen Studien abgeleitet werden kann (Mathie et al. 2007), ist aus den bisherigen Untersuchungen kein zwingender und eindeutiger Zusammenhang nachweisbar (Mayer et al. 2010). Eine eindeutige Trennung zwischen den Auswirkungen des Klimawandels und den Konsequenzen menschlicher Eingriffe lässt sich momentan noch nicht direkt und gesichert ableiten.

\subsection{Kryosphäre}

Naturgefahren in der Kryosphäre - in Gebieten mit gefrorenem Wasser - sind ganz unterschiedlich in ihrer räumlichen Verbreitung, in ihrer zeitlichen Aktivität und in Bezug auf ihre Wechselwirkung mit der Gesellschaft zu betrachten (Damm et al. 2012). Jedoch greift der Mensch in die Kryosphäre weniger ein, sodass die Auswirkungen seines Handelns auf diese Naturgefahren nicht so stark sind wie auf die Bereiche der gravitativen Massenbewegungen oder der Waldbrände. Dieser Beitrag konzentriert sich auf die Naturgefahren, ausgelöst durch den flächenhaften Rückgang des Permafrosts, durch Veränderungen von glazialen Systemen und Schneelawinen (Haeberli und Beniston 1998).

\subsubsection{Auftauender Permafrost}

Dauergefrorener Boden und Fels unterliegen momentan global massiven Veränderungen (Kääb 2007). Auch in Deutschland werden - wenn auch nur in Hochgebirgsregionen - seit einigen Jahren signifikante Veränderungen dokumentiert (Krautblatter et al. 2010a), die sicherlich auch mit dem Klimawandel in Verbindung stehen. Der Anstieg der durchschnittlichen Jahrestemperatur und die damit verbundene Erhöhung der Null-GradIsotherme (Linie gleicher Temperaturen) im Hochgebirge führen dazu, dass sich der Permafrost kontinuierlich abbaut (Gude und Barsch 2005).

Wie bei den Felsstürzen und den Muren bereits ausgeführt, kann erwartet werden, dass der Rückgang des Permafrosts massive Veränderungen im Prozessgefüge und in der Dynamik der Naturgefahren bewirkt (Damm und Felderer 2013). In den Regionen mit steilen Felswänden ist bereits zu beobachten, dass die Felssturzaktivität steigt (Krautblatter et al. 2010a). Durch den verschwindenden Permafrost tauen ganze Bergregionen auf, was besonders große Auswirkungen auf die dort vorhandene Infrastruktur hat, seien es die Bergbahnen mit den Bergstationen für den Tourismus, das Observatorium der Zugspitze oder die bewirtschafteten Berghütten der Alpenvereine (Weber 2003; Gude und Barsch 2005; Krautblatter et al. 2010b). Auch hochgelegene Schutthalden und Moränenzüge wurden bisher durch den Permafrost stabilisiert. Durch das Auftauen des gefrorenen Schutts kann dieser bei Starkniederschlägen leichter mobilisiert werden, und es besteht die Gefahr von häufigeren und größeren Murabgängen (Damm und Felderer 2013).

Neben diesen klassischen Naturgefahren verändert sich durch eine Klimaerwärmung auch das komplette Prozessgefüge in Hochgebirgsgebieten, die zwar unvergletschert, aber dennoch durch Frost geprägt sind. Es kann erwartet werden, dass sich die Solifluktion - die fließende Bewegung von Schutt- und Erdmassen an Hängen auf gefrorenem Untergrund - mit dem auftauenden Permafrost durch die erhöhte Wasserverfügbarkeit zuerst auf Bewegungsraten von bis zu mehreren Zentimetern bis Metern pro Jahr verstärkt, dann aber durch das fehlende Wasser wieder stark auf Millimeter bis Zentimeter pro Jahr reduzieren wird. Der Eisanteil in aktiven Blockgletschern kann stark abnehmen. Durch das Verschwinden des Eisanteils wird sich die interne Reibung der Schutt- und Geröllmasse kontinuierlich 
erhöhen, bis sich diese nicht weiter bewegen werden. All diese Veränderungen werden $u$.a. die Oberflächenprozesse in ihren Eigenschaften und in ihrem räumlichen und zeitlichen Auftreten nachhaltig modifizieren.

\subsubsection{Glaziale Systeme}

Bereits seit vielen Jahren wird beobachtet, dass die glazialen Systeme global einer großen Veränderung unterliegen, was in den meisten Fällen einen massiven Gletscherrückzug bedeutet (Weber 2003; Owen et al. 2009; Zemp et al. 2006). Viele Studien zeigen, dass auch die in Deutschland befindlichen Gletscher an Masse verlieren und sich zurückziehen (Haeberli und Beniston 1998; Weber 2003). Dieser Trend wird sich in den kommenden Jahren noch fortsetzen, und es ist bei einer anhaltenden Klimaerwärmung bis zum Ende des 21. Jahrhunderts sogar zu erwarten, dass auch die letzten Gletscher in Deutschland bald verschwunden sein werden.

Dies wird signifikante Auswirkungen in den hochalpinen Gebieten, aber auch in den glazial geprägten Flusssystemen haben. Momentan ist in den europäischen Alpen zu beobachten, dass durch die erhöhten Schmelzraten im Sommer die Wasserverfügbarkeit bedeutend steigt und deshalb die sommerliche Wasserführung in den glazialen Flussregimen zunimmt (Collins 2007). Hierdurch nehmen die Sedimentfrachten in den Flüssen zu. Es ist jedoch zu erwarten, dass sich diese erhöhte Wasserführung mit dem Abschmelzen der Gletscher umgehend vermindert, wie dies bereits in anderen Regionen festgestellt wird (u. a. in Chile, Baraer et al. 2012). Wahrscheinlich wird sich das Abflussregime von einem glazialen Regime mit sommerlichen Abflussspitzen zu einem schneegeprägten Abflussregime mit Spitzen im Frühjahr verändern. Dies wird sicherlich massive Auswirkungen auf das komplette hochalpine Ökosystem haben, aber auch das raumwirksame Handeln der Menschen in den Tallagen der Gebirge verändern. Besonders ist hier zu beachten, dass diese Veränderungen in den gesamten Alpen stattfinden. Für Deutschland bedeutet dies, dass sich auch Flusssysteme, die ihr Quellgebiet in den an Deutschland angrenzenden alpinen Gebieten haben (z. B. in Österreich und der Schweiz), stark verändern werden.

Auch das von Gletschern frei werdende Gebiet wird sich massiv wandeln. Es beginnen periglaziale Prozesse in den bisher durch Eis bedeckten Regionen. Flächenmäßig sind dies, besonders in der Relation der gesamten Bundesrepublik, nur marginale Flächen. Diese werden sich jedoch signifikant verändern.

\subsubsection{Schneelawinen}

Mit der gemessenen Erwärmung steigt die Null-Grad-Isotherme in den Hochgebirgen, und es ist zu erwarten, dass sich der Anteil des als Schnee fallenden Niederschlags in Zukunft zugunsten des Anteils von in flüssiger Form fallendem Niederschlag verschiebt. Die Erhöhung der Schneegrenze wird dazu führen, dass weniger Schnee zur Verfügung steht. Dies wird auch einen Einfluss auf den Schneedeckenaufbau haben, da in höheren Lagen aufgrund der veränderten Gegensätze der Tag-/Nacht-Temperaturen die Anzahl der Frost-Tau-Zyklen steigen wird und somit eine stärkere Schichtung der Schneedecke mit verändertem Wasserhaushalt zu erwarten ist (Bernhardt et al. 2012; Steinkogler et al. 2014).

Neben der Schneedecke selbst sind gerade für Schneelawinen die Schneeakkumulationen durch Windverfrachtung von zentraler Bedeutung (Warscher et al. 2013). Inwieweit sich mit der Klimaerwärmung auch Windfelder und die Verteilung der winterlichen Schneeakkumulationen ändern werden, ist schwer zu beurteilen. Weiterhin wird sicherlich weniger Schnee in tiefen Lagen abgelagert (Eckert et al. 2010; Lavigne et al. 2015). Es ist aber auch zu erwarten, dass Extremereignisse große Schneemengen in die Hänge bringen und, kombiniert mit schnellen Wetteränderungen, in kurzen Perioden die Schneelawinenaktivität erhöhen (Pielmeier et al. 2013). Zusätzlich könnte die Schneelawinenaktivität über den ganzen Winter verteilt eher abnehmen, Extremniederschlagsereignisse mit entsprechenden Lawinenabgängen wird es aber durchaus weiter geben.

\subsection{Ausblick}

Man muss klimarelevante Naturgefahren sehr differenziert betrachten. Einfache Kausalschlüsse zwischen Klimaveränderungen und natürlichen Prozessen an der Erdoberfläche können irreführend sein. Das Auftreten der präsentierten Naturgefahren ist von den vorbereitenden, auslösenden und kontrollierenden Faktoren abhängig. Wie dargelegt, unterscheidet sich die Bedeutung der jeweiligen Faktoren für die verschiedenen Naturgefahren signifikant. Zusätzlich wird die Einschätzung der Situation noch erschwert, da auch der Mensch direkt oder indirekt massiv in die Umwelt eingreift (Birkmann et al. 2011). Dadurch verändern sich die Wirkungsketten bei den jeweiligen Naturgefahren und somit auch die Konsequenzen (Klose et al. 2012). Diese lassen sich dadurch schwerer von den aus dem Klimawandel resultierenden Kräften differenzieren.

Um diese Aspekte in der Zukunft umfassend und im Sinne eines besseren Verständnisses der möglichen Auswirkungen des Klimawandels auch hinsichtlich einer Nachhaltigkeit besser verstehen zu können, sollten einige der angesprochenen Themenkomplexe bearbeitet werden. Neben vielen anderen Themen beinhaltet dies Folgendes:

- Die vielfältigen Wechselwirkungen der klimatologischen und hydrometeorologischen Faktoren müssen prozessorientiert durch Geländeuntersuchungen und ergänzende Modellierungen aufgearbeitet werden.

- Die vergangenen Situationen müssen den momentanen Gegebenheiten und den möglichen zukünftigen Entwicklungen gegenübergestellt werden.

- In Prozessuntersuchungen muss eindeutig zwischen vorbereitenden, auslösenden und kontrollierenden Faktoren unterschieden werden. Dies wird eine bessere Abschätzung der Auswirkungen der Änderungen im Klimasystem bei den verschiedenen Naturgefahren erlauben. Spezifisch für jede Naturgefahr müssen die möglichen menschlichen Eingriffe identifiziert und ihre Bedeutung in der jeweiligen Kinematik abgeschätzt und kalkuliert werden. 
- Die natürlichen und die menschlichen Eingriffe müssen vergleichend bewertet werden, um die Auswirkungen der Änderungen einzelner Faktoren für spezifische Naturgefahren eindeutig abschätzen zu können.

- Die Kaskadeneffekte zwischen den einzelnen Naturgefahren müssen stärker berücksichtigt werden. Beispielsweise können ein Waldbrand oder eine Schneelawine dazu führen, dass in der darauf folgenden Zeit Felsstürze in tiefer gelegene Gebiete gelangen können, da die frühere Schutzwirkung des Waldes entfällt. Oder Muren können Flüsse blockieren: Es bilden sich Seen, die dann den Damm durchbrechen und große Überschwemmungen in den talabwärtsgelegenen Gebieten verursachen können.

\subsection{Kurz gesagt}

Klimarelevante Naturgefahren sind auf vielfältige Faktoren zurückzuführen, deren Zusammenwirken in der Gesamtheit betrachtet werden muss. Die vorbereitenden, auslösenden und kontrollierenden Faktoren werden in unterschiedlichster Weise vom Klimawandel beeinflusst. Dieses Zusammenspiel zeigt sich durch schleichende Veränderungen wie bei Dürre, Rückgang des Permafrosts und kriechenden gravitativen Massenbewegungen sowie an schnell ablaufenden Naturgefahren wie Waldbränden, Muren, Fels- und Bergstürzen sowie Schneelawinen. Klimatische und hydrometeorologische Faktoren beeinflussen hierbei die Naturgefahren langfristig auch überregional, z. B. die Auswirkungen lang anhaltender Dürre. Sie bestimmen aber auch ganz kurzfristig in kleinen Gebieten entsprechende Prozesse, etwa Muren nach einem Starkniederschlagsereignis. Weiterhin erschwert der menschliche Einfluss auf natürliche Prozesse die klare Zuordnung, welche der Veränderungen in der Häufigkeit oder der Stärke von Naturgefahren tatsächlich ausschließlich dem Klimawandel zuzuschreiben sind und welche Anteile hierbei der direkte menschliche Einfluss hat (z.B. besonders bei Waldbränden). Dies werden einige der zukünftigen Forschungsfelder im Kontext der klimarelevanten Naturgefahren ergründen.

Dürren, Waldbrände, gravitative Massenbewegungen und andere klimarelevante Naturgefahren lassen sich zwar auf den Klimawandel zurückführen, dürfen aber auch nicht darauf reduziert werden. Es gibt neben den klimatischen Steuerungen noch viele weitere, vom Klima nicht direkt beeinflusste Faktoren, die diese Naturgefahren sehr stark beeinflussen und sich erschwerend auch noch mit den Klimaveränderungen überlagern.

\section{Literatur}

Amatulli G, Camia A, San-Miguel-Ayanz J (2013) Estimating future burned areas under changing climate in the EU-Mediterranean countries. Sci Total Environ 450:209-222. doi:10.1016/j.scitotenv.2013.02.014

Andrecs P, Hagen K, Lang E, Stary U, Gartner K, Herzberger E, Riedel F, Haiden T (2007) Dokumentation und Analyse der Schadensereignisse 2005 in den Gemeinden Gasen und Haslau (Steiermark). BFW-Dokumentation. Schriftenreihe des Bundesforschungs- und Ausbildungszentrums für Wald, Naturgefahren und Landschaft, Bd. 6. Bundesforschungs- und Ausbildungszentrum für Wald, Naturgefahren und Landschaft, Wien, S 75
Badeck F-W, Lasch P, Hauf Y, Rock J, Suckow F, Thonicke K (2004) Steigendes klimatisches Waldbrandrisiko. AFZ/Wald 59(2):90-93

Baraer M, Mark BG, McKenzie JM, Condom T, Bury J, Huh K-I, Portocarrero C, Gomez J, Rathay S (2012) Glacier recession and water resources in Peru's Cordillera Blanca. J Glaciol 58(207):134-150. doi:10.3189/2012JoG11J1

Bedia J, Herrera S, San Martin D, Koutsias N, Gutierrez JM (2013) Robust projections of Fire Weather Index in the Mediterranean using statistical downscaling. Clim Chang 120(1-2):229-247. doi:10.1007/s10584-013-0787-3

Bernhardt M, Schulz K, Liston GE, Zängl G (2012) The influence of lateral snow redistribution processes on snow melt and sublimation in alpine regions. J Hydrol 424-425:196-206

Birkmann J, Böhm HR, Buchholz F, Büscher D, Daschkeit A, Ebert S, Fleischhauer M, Frommer B, Kähler S, Kufeld W, Lenz S, Overbeck G, Schanze J, Schlipf S, Sommerfeldt P, Stock M, Vollmer M, Walkenhorst O (2011) Glossar Klimawandel und Raumplanung Bd. 10. Akademie für Raumforschung und Landesplanung, Hannover (E-Paper der ARL)

BLE (2011) Waldbrandstatistik der Bundesrepublik Deutschland. Bundesanstalt für Landwirtschaft und Ernährung

Bock B, Wehinger A, Krauter E (2013) Hanginstabilitäten in Rheinland-Pfalz Auswertung der Rutschungsdatenbank Rheinland-Pfalz für die Testgebiete Wißberg, Lauterecken und Mittelmosel. Mainzer Geowiss Mitt 41:103-122

Cane D, Wastl C, Barbarino S, Renier L A, Schunk C, Menzel A (2013) Projection of fire potential to future climate scenarios in the Alpine area: some methodological considerations. Climatic Change August 2013, Volume 119, Issue 3, pp 733-746

Climate Explorer (http://climexp.knmi.nl/)18.08.16

Collins DN (2007) Changes in quantity and variability of runoff from Alpine basins with climatic fluctuation and glacier decline. IAHS Publ 318:75-86

Damm B, Felderer A (2013) Impact of atmospheric warming on permafrost degradation and debris flow initiation - a case study from the eastern European Alps. E\&G Quaternary Science Journal 62:2

Damm B, Pröbstl U, Felderer A (2012) Perception and impact of natural hazards as consequence of warming of the cryosphere in tourism destinations. $A$ case study in the Tux Valley, Zillertaler Alps, Austria. Interpraevent 12:90-91

Dehn M, Buma J (1999) Modelling future landslide activity based on general circulation models. Geomorphology 30(1-2):175-187

Dikau R, Schrott L (1999) The temporal stability and activity of landslides in Europe with respect to climatic change (TESLEC): main objectives and results. Geomorphology 30:1-12

DWD (2004) Klimastatusbericht 2003: Statistisch-klimatologische Analyse des Hitzesommers 2003 in Deutschland. Deutscher Wetterdienst, Offenbach, S $123-132$

DWD (2010) Klimastatusbericht 2009. Deutscher Wetterdienst, Offenbach

DWD (2014) Deutscher Klimaatlas. http://www.dwd.de/DE/klimaumwelt/klimaatlas/klimaatlas_node.html. Zugegriffen: 11. Mai 2016

Eckert N, Baya H, Deschatres M (2010) Assessing the response of snow avalanche runout altitudes to climate fluctuations using hierarchical modeling: Application to 61 winters of data in France. J Clim 23:3157-3180. doi:10.1175/2010JCLI3312.1

Finkler C, Emde K, Vött A (2013) Gravitative Massenbewegungen im Randbereich des Mainzer Beckens: Das Fallbeispiel Roterberg (Langenlonsheim, Rheinland-Pfalz). Mainzer Geowiss Mitt 41:51-102

Fischer EM, Schär C (2010) Consistent geographical patterns of changes in highimpact European heatwaves. Nat Geosci. doi:10.1038/NGEO866

García A, Hördt A, Fabian M (2010) Landslide monitoring with high resolution tilt measurements at the Dollendorfer Hardt landslide, Germany. Geomorphology 120(1-2):16-25

Glade T, Dikau R (2001) Landslides at the tertiary escarpments in Rheinhessen, Southwest Germany. Z Geomorphol, (Supplementband) 125:65-92

Glade T, Anderson M, Crozier MJ (Hrsg) (2005) Landslide hazard and risk. Wiley, Chichester, West Sussex, $\mathrm{S} 803$

Gude M, Barsch D (2005) Assessment of geomorphic hazards in connection with permafrost occurrence in the Zugspitze area (Bavarian Alps, Germany). Geomorphology 66(1-4):85-93

Günther A, Thiel C (2009) Combined rock slope stability and shallow landslide susceptibility assessment of the Jasmund cliff area (Rügen Island, Germany). Nat Hazards Earth Syst Sci 9(3):687-698 
Haas F, Heckmann T, Klein T, Becht M (2009) Rockfall measurements in Alpine catchments (Germany, Austria, Italy) by terrestrial laserscanning first results. Geophys Res Abstr 11:1607-7962

Haeberli W, Beniston M (1998) Climate Change and its impacts on glaciers and permafrost in the Alps. Ambio 27(4):258-265

Hardenbicker U, Halle S, Grunert JM (2001) Temporal occurrence of mass movements in the Bonn area. Z Geomorphol, Supplementband 125:14-24

Hattermann FF, Kundzewicz ZW, Huang S, Vetter T, Gerstengarbe FW, Werner PC (2013) Climatological drivers of changes in flood hazard in Germany. Acta Geophys 61(2):463-477. doi:10.2478/s11600-012-oo70-4

IPCC (2013) Climate change 2013: The physical science basis, contribution of working group I to the fifth assessment report of the Intergovernmental Panel on Climate Change., http://www.ipcc.ch, S 1-2216

Jacob D, Petersen J, Eggert B, Alias A, Christensen O-B, Bouwer L, Braun A, Colette A, Déqué $M$, Georgievski G, Georgopoulou E, Gobiet A, Menut L, Nikulin G, Haensler A, Hempelmann N, Jones C, Keuler K, Kovats S, Kröner N, Kotlarski S, Kriegsmann A, Martin E, Meijgaard E, Moseley C, Pfeifer S, Preuschmann S, Radermacher C, Radtke K, Rechid D, Rounsevell M, Samuelsson P, Somot S, Soussana J-F, Teichmann C, Valentini R, Vautard R, Weber B, Yiou P (2013) EURO-CORDEX: new high-resolution climate change projections for European impact research. Reg Environ Change. Springer, Berlin, S 1-16

Kääb AFP (2007) Climate change impacts on mountain glaciers and permafrost. Glob Planet Chang 56:vii-ix

Klose M, Damm B, Terhorst B, Schulz N, Gerold G (2012) Wirtschaftliche Schäden durch gravitative Massenbewegungen. Entwicklung eines empirischen Berechnungsmodells mit regionaler Anwendung. Interpraevent 12:979-990

Klose M, Damm B, Terhorst B (2015) Landslide cost modeling for transportation infrastructures: a methodological approach. Landslide 12:321-334

Krautblatter M, Moser M (2006) Will we face an increase in hazardous secondary rockfall events in response to global warming in the foreseeable future? In: Price MF (Hrsg) Global change in mountain regions. Sapiens Publishing, Duncow, S 253-254

Krautblatter M, Moser M (2009) A nonlinear model coupling rockfall and rainfall intensity based \newline on a four year measurement in a high Alpine rock wall (Reintal, German Alps). Nat Hazards Earth Syst Sci 9(4):1425-1432. doi:10.5194/nhess-9-1425-2009

Krautblatter M, Moser M, Kemna A, Verleysdonk S, Funk D, Dräbing D (2010a) Climate change and enhanced rockfall activity in the European Alps. Z Dtsch Ges Geowiss 68:331-332

Krautblatter M, Verleysdonk S, Flores-Orozco A, Kemna A (2010b) Temperaturecalibrated imaging of seasonal changes in permafrost rock walls by quantitative electrical resistivity tomography (Zugspitze, German/Austrian Alps) J Geophys Res 115(F2):F02003. doi:10.1029/2008jf001209

Krautblatter M, Funk D, Günzel FK (2013) Why permafrost rocks become unstable: a rock-ice-mechanical model in time and space. Earth Surf Process Landf 38(8):876-887. doi:10.1002/esp.3374

Krauter E, Kumerics C, Feuerbach J, Lauterbach M (2012) Abschätzung der Risiken von Hang- und Böschungsrutschungen durch die Zunahme von Extremwetterereignissen. Berichte der Bundesanstalt für Straßenwesen, Straßenbau (Heft 75, 61 S)

Krysanova V, Vetter T, Hattermann F (2008) Detection of change in the drought frequency in the Elbe basin: comparison of three methods. Hydrol Sci J 53(3):519-537

Kuhn D, Prüfer S (2014) Coastal cliff monitoring and analysis of mass wasting processes with the application of terrestrial laser scanning: A case study of Rügen, Germany. Geomorphology 213:153-165

Kurdal S, Wehinger A, Krajewski W (2006) Entwicklung von Baugebieten in Rheinhessen/Rheinland-Pfalz bei möglicher Hangrutschgefährdung. Mainzer Geowiss Mitt 34:135-152

Lavalle C, Micale F, Houston TD, Camia A, Hiederer R, Lazar C, Genovese G (2009) Climate change in Europe. 3. Impact on agriculture and forestry. A review (Reprinted). Agron Sustainable Dev 29(3):433-446. doi:10.1051/ agro/2008068

Lavigne A, Eckert N, Bel L, Parent E (2015) Adding expert contributions to the spatiotemporal modelling of avalanche activity under different climatic influences. J R Stat Soc: Series C (Applied Statistics) 64(4):651-671. doi: $10.1111 /$ rssc. 12095
Masato G, Hoskins BJ, Woollings T (2013) Winter and summer Northern Hemisphere blocking in CMIP5 models. J Clim 26:7044-7059. doi:10.1175/ JCLI-D-12-00466.1

Mathie E, McInnes R, Fairbank H, Jakeways J (2007) Landslides and climate change: Challenges and solutions. proceedings of the International Conference on Landslides and Climate Change, Ventnor, Isle of Wight, UK, 21.-24.05.2007.

Mayer K, Patula S, Krapp M, Leppig B, Thom P, von Poschinger A (2010) Danger Map for the Bavarian Alps. Z Dtsch Ges Geowiss 161(2):119-128

Migliavacca M, Dosio A, Kloster S, Ward DS, Camia A, Houborg R, Cescatti A (2013a) Modeling burned area in Europe with the Community Land Model. J Geophys Res: Biogeosci 118(1):265-279. doi:10.1002/jgrg.20026

Migliavacca M, Dosio A, Camia A, Houborg R, Houston-Durrant T, Kaiser J W, Khabarov N, Krasovskii A, San Miguel-Ayanz J, Ward D S, Cescatti A (2013b) Modeling biomass burning and related carbon emissions during the 21st century in Europe. J Geophys Res: Biogeosci 118(4):1732-1747. doi:10.1002/2013JG002444

Müller M (2009) Auswirkungen des Klimawandels auf ausgewählte Schadfaktoren in den deutschen Wäldern. Wiss Zeitschr d TU Dresden 58(3-4):69-75

Münchner Rückversicherungs-Gesellschaft (2012) Georisikoforschung NatCatSERVICE (Stand Juli 2012)

Oeltzschner H (1997) Untersuchungen von Massenbewegungen im südlichen Bayern durch das Bayerische Geologische Landesamt. Wasser Boden 49(1):46-50. doi:10.1016/j.geomorph.2008.04.019

Owen LA, Thackray G, Anderson RS, Briner J, Kaufman D, Roe G, Pfeffer W, YiC (2009) Integrated research on mountain glaciers: Current status, priorities and future prospects. Geomorphology 103(2):158-171. doi:10.1016/j.geomorph.2008.04.019

Papathoma-Köhle M, Glade T (2013) The role of vegetation cover change for landslide hazard and risk. In: Renaud G, Sudmeier-Rieux K, Estrella M (Hrsg) The role of ecosystems in disaster risk reduction. UNU-Press, Tokio, S 293-320

Petoukhov V, Rahmstorf S, Petri S, Schellnhuber HJ (2013) Quasiresonant amplification of planetary waves and recent Northern hemisphere weather extremes. Proceedings of the National Academy of Science of the USA. doi:10.1073/pnas.1222000110

Pielmeier C, Techel F, Marty C, Stucki T (2013) Wet snow avalanche activity in the Swiss Alps - trend analysis for mid-winter season. In: Naaim-Bouvet F, Durand Y, Lambert R (Hrsg) International Snow Science Workshop 2013 Proceedings. ISSW 2013, Grenoble - Chamonix Mont Blanc, 07.-11.10.2013. ANENA, Grenoble, S 1240-1246

Röhlich B, Jehle R, Krauter E (2003) Systematische Bestandsaufnahme des Gefährdungspotentials an Bahnstrecken durch Steinschlag, Felssturz und Hangrutsch im Mittelrhein-, Mosel- und Lahngebiet. 14. Tagung für Ingenieurgeologie, Kiel, 26.-29.03.2003., S 281-286

San-Miguel-Ayanz J, Moreno JM, Camia A (2013) Analysis of large fires in European Mediterranean landscapes: Lessons learned and perspectives. Forest Ecology and Management 294:11-22

Schär C, Vidale PL, Lüthi D, Frei C, Häberli C, Liniger M, Appenzeller C (2004) The role of increasing temperature variability in European summer heat waves. Nature 427:332-336

Schmidt J, Dikau R (2004) Modeling historical climate variability and slope stability. Geomorphology 60(3-4):433-447

Schmidt J, Dikau R (2005) Preparatory and triggering factors for slope failure: Analyses of two landslides near Bonn, Germany. Z Geomorphol 49(1):121138

Schmidt KH, Beyer I (2001) Factors controlling mass movement susceptibility on the Wellenkalk-scarp in Hesse and Thuringia. Z Geomorphol, (Supplementband) 125:43-63

Schneider H, Höfer D, Irmler R, Daut G, Mäusbacher R (2010) Correlation between climate, man and debris flow events - A palynological approach. Geomorphology 120(1):48-55

Schönwiese CD, Janoschitz R (2005) Klima-Trendatlas Deutschland 1901-2000 Bd. 4. Inst. Atmosphäre Umwelt, Univ., Frankfurt/Main

Steinkogler W, Sovilla B, Lehning M (2014) Influence of snow cover properties on avalanche dynamics. Cold Reg Sci Technol 97:121-131 doi: 10.1016/j. coldregions.2013.10.002 
Terhorst B (2001) Mass movements of various ages on the Swabian Jurassic escarpment geomorphologic processes and their causes. Z Geomorphol 125, (Supplementband 125):105-127

Terhorst B (2009) Landslide susceptibility in cuesta scarps of SW-Germany (Swabian Alb). In: Bierman P, Montgomery P (Hrsg) Key concepts in geomorphology

Thonicke K, Cramer W (2006) Long-term trends in vegetation dynamics and forest fires in Brandenburg (Germany) under a changing climate. Natural Hazards 38(1):

Wagner CE van (1987) Development and structure of the Canadian Forest Fire Index. Canadian Forestry Service, Technical Report 35: S 37

Warscher M, Strasser U, Kraller G, Marke T, Franz H, Kunstmann H (2013) Performance of complex snow cover descriptions in a distributed hydrological model system: A case study for the high Alpine terrain of the Berchtesgaden Alps. Water Resour Res 49(5):2619-2637. doi: 10.1002/wrcr.20219

Wastl C, Schunk C, Leuchner M, Pezzatti GB, Menzel A (2012) Recent climate change: Long-term trends in meteorological forest fire danger in the Alps. Agric For Meteorol 162:1-13. doi:10.1016/j.agrformet.2012.04.001

Weber M (2003) Informationen zum Gletscherschwund - Gletscherschwund und Klimawandel an der Zugspitze und am Vernagtferner (Ötztaler Alpen). Kommission für Glaziologie der Bayerischen Akademie der Wissenschaften, S 10

Wittich K-P, Löpmeier F-J, Lex P (2011) Waldbrände und Klimawandel in Deutschland. AFZ/Wald 18:22-25

WMO (2012) MO statement on the status of the global climate in 2011. http:// www.wmo.int/pages/prog/wcp/wcdmp/documents/1085_en.pdf. Zugegriffen: 15. Mai 2016

WMO (2014) WMO statement on the status of the global climate in 2013. https://drive.google.com/file/d/OBwdvoC9AeWjUeEV1cnZ6QURVaEE/ edit?pref=2\&pli=1. Zugegriffen: 11. Mai 2016

Zemp M, Haeberli W, Hoelzle M, Paul F (2006) Alpine glaciers to disappear within decades? Geophys Res Lett 33(13):L13504. doi:10.1029/2006gl026319

Open Access Dieses Kapitel wird unter der Creative Commons Namensnennung 4.0 International Lizenz (http://creativecommons.org/ licenses/by/4.0/deed.de) veröffentlicht, welche die Nutzung, Vervielfältigung, Bearbeitung, Verbreitung und Wiedergabe in jeglichem Medium und Format erlaubt, sofern Sie den/die ursprünglichen Autor(en) und die Quelle ordnungsgemäß nennen, einen Link zur Creative Commons Lizenz beifügen und angeben, ob Änderungen vorgenommen wurden.

Etwaige Abbildungen oder sonstiges Drittmaterial unterliegen ebenfalls der genannten Creative Commons Lizenz, sofern sich aus der Abbildungslegende oder der Quellreferenz nichts anderes ergibt. Sofern solches Drittmaterial nicht unter der genannten Creative Commons Lizenz steht, ist eine Vervielfältigung, Bearbeitung oder öffentliche Wiedergabe nur mit vorheriger Zustimmung des betreffenden Rechteinhabers oder auf der Grundlage einschlägiger gesetzlicher Erlaubnisvorschriften zulässig. 